\title{
Hamiltonian Cycles in the Square of a Graph
}

\author{
Jan Ekstein* \\ Department of Mathematics \\ University of West Bohemia \\ Pilsen, Czech Republic \\ ekstein@kma.zcu.cz
}

Submitted: Oct 6, 2009; Accepted: Oct 6, 2011; Published: Oct 17, 2011

Mathematics Subject Classifications: 05C45

\begin{abstract}
We show that under certain conditions the square of the graph obtained by identifying a vertex in two graphs with hamiltonian square is also hamiltonian. Using this result, we prove necessary and sufficient conditions for hamiltonicity of the square of a connected graph such that every vertex of degree at least three in a block graph corresponds to a cut vertex and any two these vertices are at distance at least four.
\end{abstract}

Keywords: hamiltonian cycle; connection of graphs; block graph; square; star

\section{Introduction and notation}

The graphs considered in this paper are undirected and simple. If $G$ is a graph, we denote by $V(G)$ the vertex set of $G$, by $E(G)$ the edge set of $G$. For $x \in V(G), d_{G}(x)$ denotes the degree of $x$ and $N_{G}(x)$ denotes the neighbourhood of $x$. For $x, y \in V(G)$, $\operatorname{dist}_{G}(x, y)$ denotes the distance between $x, y$. For $A \subseteq V(G),\langle A\rangle$ denotes the subgraph of $G$ induced by $A$.

The $k$-star is a tree on $k+1$ vertices with one vertex of degree $k$, called the center, and the others of degree $1, k=0,1,2, \ldots$. The graph $S\left(K_{1,3}\right)$ is the graph $K_{1,3}$ in which each edge is subdivided once. Given sets $A, B$ of vertices, we call $P=x_{0}, \ldots, x_{k}$ an $(A, B)$-path if $V(P) \cap A=\left\{x_{0}\right\}$ and $V(P) \cap B=\left\{x_{k}\right\}$, we write $(a, B)$-path rather than $(\{a\}, B)$-path. For a graph $G$ we define $V_{i}(G)=\{v \in V(G): d(v)=i\}$ and $W(G)=V(G) \backslash V_{2}(G)$. A branch in $G$ is a nontrivial path whose ends are in $W(G)$ and whose internal vertices, if any, are of degree 2 in $G$.

\footnotetext{
*Supported by the grant 1M0545 of the Czech Ministry of Education.
} 
The square of $G$, denoted $G^{2}$, is the graph with the vertex set $V(G)$ in which two vertices are adjacent if their distance in $G$ is one or two. We say that two graphs are homeomorphic if they can be turned into isomorphic graphs by finite number of edgesubdivisions. Let $G^{\prime}$ be an subgraph of $G$. We say that $G^{\prime}$ is maximal with respect to a given graph property if $G^{\prime}$ itself has the property but no graph $G^{\prime}+A$ does, for any nonempty subset $A \subseteq E(G) \backslash E\left(G^{\prime}\right)$.

A connected graph that has no cut vertices is called a block. A block of a graph is a subgraph that is a block and is maximal with respect to this property. The degree of a block $B$ of a graph $G$, denoted by $d(B)$, is the number of cut vertices of $G$ belonging to $V(B)$. A block of degree 1 is called an endblock of $G$, otherwise it is a non-end block. A block is said to be acyclic if it is isomorphic to one edge, otherwise we say it is cyclic. The block graph of a graph $G$ is the graph $\operatorname{Bl}(G)$ such that the vertices of $\operatorname{Bl}(G)$ are the blocks and cut vertices of $G$, and two vertices are adjacent in $\operatorname{Bl}(G)$ if one of them is a block of $G$ and the second one is its vertex.

Let $G_{1}, G_{2}$ be connected graphs, $x \notin V\left(G_{1}\right) \cup V\left(G_{2}\right), V\left(G_{1}\right) \cap V\left(G_{2}\right)=\emptyset$, and let $x_{i} \in V\left(G_{i}\right), i=1,2$. Then the graph $G$ with vertex set $V(G)=\left(V\left(G_{1}\right) \backslash\left\{x_{1}\right\}\right) \bigcup$ $\left(V\left(G_{2}\right) \backslash\left\{x_{2}\right\}\right) \bigcup\{x\}$ and with edge set $E(G)=E\left(G_{1}-x_{1}\right) \bigcup E\left(G_{2}-x_{2}\right) \bigcup$ $\left\{u x \mid u \in V\left(G_{1}\right), u x_{1} \in E\left(G_{1}\right)\right\} \bigcup\left\{v x \mid v \in V\left(G_{2}\right), v x_{2} \in E\left(G_{2}\right)\right\}$ is called the connection of the graphs $G_{1}, G_{2}$ over the vertices $x_{1}, x_{2}$, denoted $G=G_{1}\left[x_{1}=x_{2}\right] G_{2}$.

Let $G$ be a connected graph such that $G^{2}$ is hamiltonian and let $x \in V(G)$. We say that

a) the vertex $x$ is of type 1 if there exists a hamiltonian cycle $C$ of $G^{2}$ such that both edges of $C$ incident with $x$ are in $G$,

b) the vertex $x$ is of type 2 if $x$ is not of type 1 and there exists a hamiltonian cycle $C$ of $G^{2}$ such that exactly one edge of $C$ incident with $x$ is in $G$,

c) the vertex $x$ is of type 3 if $x$ is not of type 1 or 2 and there exists a hamiltonian cycle $C$ of $G^{2}$ such that for some two vertices $u, v \in N_{G}(x)$ is $u v \in E(C)$,

d) the vertex $x$ is of type 4 if $x$ is not of type 1 or 2 or 3 .

We denote $V_{[i]}(G)=\{x \in V(G) \mid x$ is of type $i\}, i=1,2,3,4$.

\section{The connection of graphs}

Let us first mention the following result by Fleischner [2] that will be used many times in proofs.

Theorem 2.1 [2]. Let $y$ and $z$ be arbitrarily chosen vertices of a 2-connected graph $G$. Then $G^{2}$ contains a hamiltonian cycle $C$ such that the edges of $C$ incident with $y$ are in $G$ and at least one of edges of $C$ incident with $z$ is in $G$. If $y$ and $z$ are adjacent in $G$, then these are three different edges.

It is easy to see that Theorem 2.1 implies that the square of a 2-connected graph is hamiltonian. 
The following result shows that, under certain conditions, the square of the connection of two graphs with hamiltonian square is also hamiltonian.

Theorem 2.2. Let $G_{1}, G_{2}$ be connected graphs such that $\left(G_{1}\right)^{2},\left(G_{2}\right)^{2}$ are hamiltonian, let $x_{i} \in V\left(G_{i}\right), i=1,2$. If

I) $G=G_{1}\left[x_{1}=x_{2}\right] G_{2}$ and $x_{i} \in V_{[1]}\left(G_{i}\right) \cup V_{[2]}\left(G_{i}\right), i=1,2$, or

II) $G=G_{1}\left[x_{1}=x_{2}\right] K_{2}, x_{1} \in V_{[1]}\left(G_{1}\right) \bigcup V_{[2]}\left(G_{1}\right) \bigcup V_{[3]}\left(G_{1}\right)$ and $V\left(K_{2}\right)=\left\{x_{2}, u\right\}$ or

III) $G=G_{1}\left[x_{1}=x_{2}\right] G_{2}, x_{1} \in V_{[3]}\left(G_{1}\right)$ and $x_{2} \in V_{[1]}\left(G_{2}\right)$, then $G^{2}$ is hamiltonian.

Moreover under the assumptions of I),

a) if $x_{i} \in V_{[1]}\left(G_{i}\right), i=1,2$, then $x=x_{1}=x_{2} \in V_{[1]}(G)$;

b) if $x_{1} \in V_{[1]}\left(G_{1}\right)$ and $x_{2} \in V_{[2]}\left(G_{2}\right)$, then $x=x_{1}=x_{2} \in V_{[2]}(G)$;

c) if $G_{2}$ is 2-connected and $x_{1} \in V_{[1]}\left(G_{1}\right) \cup V_{[2]}\left(G_{1}\right)$, then $v \in V_{[1]}(G)$ for any $v \in V\left(G_{2}\right), v \neq x_{2}$

d) if $x_{i} \in V_{[2]}\left(G_{i}\right), i=1,2$, then $x=x_{1}=x_{2} \notin V_{[1]}(G) \bigcup V_{[2]}(G)$.

Moreover under the assumptions of II),

a) if $x_{1} \in V_{[1]}\left(G_{1}\right)$, then $x=x_{1}=x_{2} \in V_{[1]}(G)$;

b) if $x_{1} \in V_{[2]}\left(G_{1}\right)$, then $x=x_{1}=x_{2} \in V_{[2]}(G)$;

c) if $x_{1} \in V_{[1]}\left(G_{1}\right) \cup V_{[2]}\left(G_{1}\right)$, then $u \in V_{[2]}(G)$.

\section{Proof.}

I) Let $x=x_{1}=x_{2}$ and let $C_{1}, C_{2}$ be hamiltonian cycles in $\left(G_{1}\right)^{2},\left(G_{2}\right)^{2}$ such that $a_{1} x, b_{1} x \in E(G)$ for $a_{1} \in N_{C_{1}}(x), b_{1} \in N_{C_{2}}(x)$, respectively. Let $a_{2} \in N_{C_{1}}(x)$, $a_{1} \neq a_{2}$, and $b_{2} \in N_{C_{2}}(x), b_{1} \neq b_{2}$, and let $P_{a_{1} a_{2}}=C_{1}-x_{1}$ and $P_{b_{2} b_{1}}=C_{2}-x_{2}$. Then $P_{a_{1} a_{2}}, P_{b_{2} b_{1}}$ are hamiltonian paths in $\left(G_{1}-x\right)^{2},\left(G_{2}-x\right)^{2}$, respectively, and the cycle $C=a_{1} P_{a_{1} a_{2}} a_{2} x b_{2} P_{b_{2} b_{1}} b_{1} a_{1}$ is a hamiltonian cycle in $G^{2}$.

a) If moreover $x_{i} \in V_{[1]}\left(G_{i}\right), i=1,2$, then we can assume that $a_{2} x, b_{2} x \in E(G)$ and therefore $x \in V_{[1]}(G)$.

b) If moreover $x_{1} \in V_{[1]}\left(G_{1}\right)$ and $x_{2} \in V_{[2]}\left(G_{2}\right)$, then we can assume that $a_{2} x \in E(G)$ and it is obvious that there is no $C_{2}$ such that $b_{2} x \in E(G)$, therefore $x \in V_{[2]}(G)$.

c) If moreover $G_{2}$ is 2-connected, then for any $v \in V\left(G_{2}\right), v \neq x_{2}$, we can assume by Theorem 2.1 without loss of generality that $c_{1} v, c_{2} v \in E\left(G_{2}\right)$, $c_{1}, c_{2} \in N_{C_{2}}(v), c_{1} \neq c_{2}$. If $v \neq b_{1}$, then $c_{1} v, c_{2} v \in E(C)$, therefore $v \in V_{[1]}(G)$. If $v=b_{1}$, then we have $v x_{2} \in V\left(G_{2}\right)$ and by Theorem 2.1 $x_{2} b_{2} \in E(G)$. Then $\widetilde{C}=a_{1} P_{a_{1} a_{2}} a_{2} x b_{1} P_{b_{1} b_{2}} b_{2} a_{1}$ is also a hamiltonian cycle in $G^{2}$ and moreover the edges of $C_{2}$ incident with $v=b_{1}$ are in $\widetilde{C}$. Therefore $v \in V_{[1]}(G)$.

d) If moreover $x_{i} \in V_{[2]}\left(G_{i}\right), i=1,2$, then it is obvious that there are no $C_{1}$, $C_{2}$ such that $a_{2} x \in E(G)$ or $b_{2} x \in E(G)$ and therefore $x \notin V_{[1]}(G) \cup V_{[2]}(G)$. 
II) Case 1: $x_{1} \in V_{[3]}\left(G_{1}\right)$.

Let $x=x_{1}=x_{2}$ and let $C_{1}$ be a hamiltonian cycle in $\left(G_{1}\right)^{2}$ such that $y w \in E\left(C_{1}\right)$ for some $y, w \in N_{G_{1}}(x)$. Let $P_{y w}=C_{1}-y w$. Then $P_{y w}$ is a hamiltonian path in $\left(G_{1}\right)^{2}$ and the cycle $C=y P_{y w} w u y$ is a hamiltonian cycle in $G^{2}$.

Case 2: $x_{1} \in V_{[1]}\left(G_{1}\right) \bigcup V_{[2]}\left(G_{1}\right)$.

Let $x=x_{1}=x_{2}$ and let $C_{1}$ be a hamiltonian cycle in $\left(G_{1}\right)^{2}$ such that $y x \in E(G)$ for $y \in N_{C_{1}}(x)$. Let $z \in N_{C_{1}}(x), z \neq y$, and let $P_{z y}=C_{1}-x_{1}$. Then $P_{y z}$ is a hamiltonian path in $\left(G_{1}-x\right)^{2}$ and the cycle $C=z P_{z y} y u x z$ is a hamiltonian cycle in $G^{2}$.

a) If moreover $x_{1} \in V_{[1]}\left(G_{1}\right)$, then we can assume that $x z \in E(G)$ and therefore $x \in V_{[1]}(G)$.

b) If moreover $x_{1} \in V_{[2]}\left(G_{1}\right)$, then it is obvious that there is no $C_{1}$ such that $x z \in E(G)$ and therefore $x \in V_{[2]}(G)$.

c) Since $u x \in E(G)$ and $N_{G}(u)=\{x\}$, it is obvious that there is no cycle $\widetilde{C}$ in $G^{2}$ such that both edges of $\widetilde{C}$ incident with $u$ are in $G$ and therefore $u \in V_{[2]}(G)$.

III) Let $x=x_{1}=x_{2}$, let $C_{1}$ be a hamiltonian cycle in $\left(G_{1}\right)^{2}$ such that $y w \in E\left(C_{1}\right)$ for some $y, w \in N_{G_{1}}(x)$ and let $C_{2}$ be a hamiltonian cycle in $\left(G_{2}\right)^{2}$ such that $a x, b x \in E(G)$ for $a, b \in N_{C_{2}}(x), a \neq b$. Let $P_{y w}=C_{1}-y w$ and $P_{a b}=C_{2}-x_{2}$. Then $P_{y w}, P_{a b}$ are hamiltonian paths in $\left(G_{1}\right)^{2},\left(G_{2}-x\right)^{2}$, respectively, and the cycle $C=w P_{w y} y a P_{a b} b w$ is a hamiltonian cycle in $G^{2}$.

\section{The hamiltonian square of a graph}

This work is motivated by the following result due to El Kadi Abderrezzak, Flandrin and Ryjáček [1].

Theorem 3.1 [1]. If $G$ is a connected graph such that every induced $S\left(K_{1,3}\right)$ has at least three edges in a block of degree at most 2 , then $G^{2}$ is hamiltonian.

The following result, originally by Thomassen [5], is a corollary of Theorem 3.1.

Theorem 3.2 [5]. If the block graph of $G$ is a path, then $G^{2}$ is hamiltonian.

Before the presentation of main results we first give the following slight strengthening of Theorem 3.2 which will be needed in our proofs.

Theorem 3.3. Let $G$ be a graph such that its block graph is a path and let $u_{1}, u_{2}$ be arbitrary vertices which are not cut vertices and are contained in different endblocks of $G$.

Then $G^{2}$ contains a hamiltonian cycle $C$ such that, for $i=1,2$, 
- if $u_{i}$ is contained in a cyclic block, then both edges of $C$ incident with $u_{i}$ are in $G$, and

- if $u_{i}$ is contained in an acyclic block, then exactly one edge of $C$ incident with $u_{i}$ is in $G$.

Proof. If $G$ is a path of length at least 2, then the theorem is obvious. Thus, suppose that $G$ contains at least one cyclic block $B_{1}$ and let $k$ denote the number of blocks of $G$.

We prove the theorem by induction on $k$.

1. Let $k=2$, let $B_{2}$ be the second block of $G$, let $x=V(G)$ be the (only) cut vertex of $G$ and let $u_{1}, u_{2}$ be arbitrary vertices such that $u_{1} \in V\left(B_{1}\right), u_{2} \in V\left(B_{2}\right)$ and $u_{1} \neq x, u_{2} \neq x$. The graph $\left(B_{1}\right)^{2}$ contains a hamiltonian cycle $C_{1}$ such that the edges of $C_{1}$ incident with $u_{1}$ are in $B_{1}$ and at least one of edges of $C_{1}$ incident with $x$ is in $B_{1}$. If $u_{1}$ and $x$ are adjacent in $G$, then these are three different edges by Theorem 2.1. Then we can assume that $x \in V_{[1]}\left(B_{1}\right) \cup V_{[2]}\left(B_{1}\right)$ and $G=B_{1}\left[x_{1}=x_{2}\right] B_{2}$, where $x_{1}$ and $x_{2}$ is the copy of $x$ in $B_{1}$ and $B_{2}$, respectively.

a) If $B_{2}$ is cyclic, then the graph $G^{2}$ contains a hamiltonian cycle $C$ such that both edges of $C$ incident with $u_{2}$ are in $G$ by Theorem 2.2 Ic) and it is obvious that we can find $C$ such that also both edges of $C$ incident with $u_{1}$ are in $G$.

b) If $B_{2}=K_{2}=x_{2} u_{2}$, then the graph $G^{2}$ contains a hamiltonian cycle $C$ such that exactly one edge of $C$ incident with $u_{2}$ is in $G$ by Theorem 2.2 IIc) and it is obvious that we can find $C$ such that both edges of $C$ incident with $u_{1}$ are in $G$.

2. Suppose the assertion is true for each graph with at most $k$ blocks, let $G$ be a graph with $k+1$ blocks such that its block graph is a path and let $u_{1}, u_{2}$ be arbitrary vertices which are not cut vertices and are contained in different endblocks of $G, k \geq 2$.

Let $B_{k+1}$ be the endblock of $G$ containing $u_{2}$. We denote $\widetilde{G}=G-V\left(B_{k+1}-x\right)$, where $x \in V\left(B_{k+1}\right)$ is a cut vertex of $G$. Then $G=\widetilde{G}\left[x_{1}=x_{2}\right] B_{k+1}$, where $x_{1}$ and $x_{2}$ is the copy of $x$ in $\widetilde{G}$ and $B_{k+1}$, respectively, and we can assume by the induction hypothesis that $\widetilde{G}$ contains a hamiltonian cycle $C_{1}$ such that if $u_{1}, x_{1}$ is contained in a cyclic block, then both edges of $C_{1}$ incident with $u_{1}, x_{1}$ are in $\widetilde{G}$, and if $u_{1}, x_{1}$ is contained in an acyclic block, then exactly one edge of $C_{1}$ incident with $u_{1}, x_{1}$ is in $\widetilde{G}$, respectively. Then we can assume that $x_{1} \in V_{[1]}(\widetilde{G}) \bigcup V_{[2]}(\widetilde{G})$.

a) If $B_{k+1}$ is cyclic, $u_{2} \in V\left(B_{k+1}\right)$ and $u_{2} \neq x_{2}$, then the graph $G^{2}$ contains a hamiltonian cycle $C$ such that both edges of $C$ incident with $u_{2}$ are in $G$ by Theorem 2.2 Ic) and it is obvious that we can find $C$ such that if $u_{1}$ is contained in a cyclic block, then both edges of $C$ incident with $u_{1}$ are in $G$, and if $u_{1}$ is contained in an acyclic block, then exactly one edge of $C$ incident with $u_{1}$ is in $G$.

b) If $B_{k+1}=K_{2}=x_{2} u_{2}$, then the graph $G^{2}$ contains a hamiltonian cycle $C$ such that exactly one edge of $C$ incident with $u_{2}$ is in $G$ by Theorem 2.2 IIc) and it is obvious that we can find $C$ such that if $u_{1}$ is contained in a cyclic block, then both edges of $C$ incident with $u_{1}$ are in $G$, and if $u_{1}$ is contained in an acyclic block, then exactly one edge of $C$ incident $u_{1}$ is in $G$. 


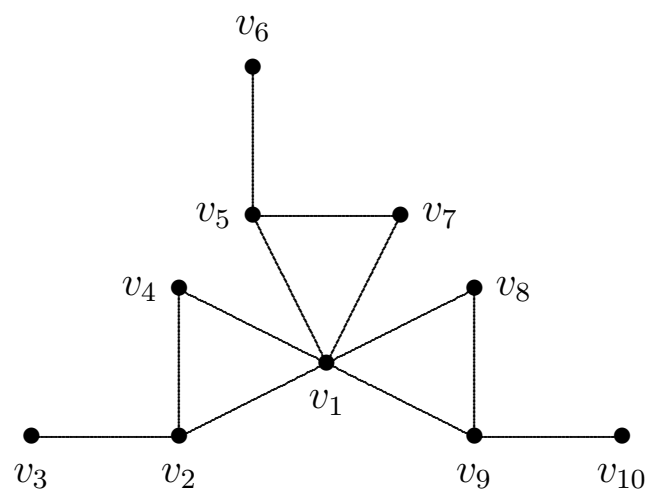

Figure 1:

We consider the graph in Figure 1. It is easy to see that the cycle $C=v_{1}, v_{2}, v_{3}$, $v_{4}, v_{5}, v_{6}, v_{7}, v_{8}, v_{9}, v_{10}, v_{1}$ is a hamiltonian cycle in $G^{2}$ but the induced subgraph $H=$ $\left\langle\left\{v_{1}, v_{2}, v_{3}, v_{5}, v_{6}, v_{9}, v_{10}\right\}\right\rangle$ is isomorphic to $S\left(K_{1,3}\right)$ and does not have at least three edges in a block of degree at most 2 . This example shows that the assumptions in Theorem 3.1 are sufficient but not necessary. We looked for other conditions implying that the square of a graph is hamiltonian.

\section{Main result}

Let $V_{\geq 3}(G)=\left\{x \in V(G) \mid d_{G}(x) \geq 3\right\}$ and, for $x \in V(G), t_{G}(x)$ denotes the number of acyclic non-end blocks of $G$ containing $x$. First of all we prove the following lemma.

Lemma 4.1. Let $G$ be a connected graph with exactly one vertex in $V_{\geq 3}(B l(G))$ corresponding to a cut vertex $a$ of $G$. If $a$ is contained in at most two acyclic non-end blocks of $G$, then $G^{2}$ contains a hamiltonian cycle $C$ such that if $t_{G}(a)=0$, then both edges of $C$ incident with $a$ are in $G$, if $t_{G}(a)=1$, then exactly one edge of $C$ incident with $a$ is in $G$, if $t_{G}(a)=2$, then no edge of $C$ incident with $a$ is in $G$.

Proof. Let $r \geq 0, s \geq 0$ and $t=t_{G}(a) \geq 0$ denote the number of cyclic blocks, acyclic endblocks and acyclic non-end blocks of $G$ containing $a$, respectively, and choose the notation such that if $r>0$, then $B_{1}, \ldots, B_{r}$ are all cyclic blocks, if $s>0$, then $B_{r+1}, \ldots, B_{r+s}$ are all acyclic endblocks, and if $t>0$, then $B_{r+s+1}, \ldots, B_{r+s+t}$ are all acyclic non-end blocks of $G$ containing the vertex $a$.

By the assumption, $t \leq 2$ and $r+s+t \geq 3$, hence $r+s>0$.

Case 1: $r=0$.

If $t=0$, then $G$ is a star and the assertion is obvious. Let $t \geq 1$.

Subcase 1.1: $s=1$. Then necessarily $t=2$. Let $B_{1}=a u$ and let $b_{2}, b_{3}$ be the branch of $\mathrm{Bl}(G)$ containing the vertex corresponding to $B_{2}, B_{3}$ and denote $H_{2}, H_{3}$ the subgraph corresponding to $b_{2}, b_{3}$, respectively. For $i=2,3, \mathrm{Bl}\left(H_{i}\right)$ is a path and therefore $\left(H_{i}\right)^{2}$ is hamiltonian and $a \in V_{[2]}\left(H_{i}\right)$ by Theorem 3.3. If $G_{1}=H_{2}\left[x_{1}=x_{2}\right] B_{1}$, where $x_{1}$ and $x_{2}$ 
is the copy of $a$ in $H_{2}$ and $B_{1}$, respectively, then $\left(G_{1}\right)^{2}$ is hamiltonian and $a \in V_{[2]}\left(G_{1}\right)$ by Theorem $2.2 \mathrm{IIb})$. Moreover $G=G_{1}\left[y_{1}=y_{2}\right] H_{3}$, where $y_{1}$ and $y_{2}$ is the copy of $a$ in $G_{1}$ and $H_{3}$, respectively, and $G^{2}$ contains hamiltonian cycle $C$ such that no edge of $C$ incident with $a$ is in $G$ by Theorem 2.2 Id).

Subcase 1.2: $s \geq 2$. Then necessarily $1 \leq t \leq 2$. For $i=s+1, s+2$, let $H_{i}$ be the same subgraphs as in Subcase 1.1 and let $\widetilde{\widetilde{G}}=G-V\left(H_{s+1}-a\right)-V\left(H_{s+2}-a\right)$. It is obvious that $\widetilde{G}$ is a star and therefore $(\widetilde{G})^{2}$ is hamiltonian and $a \in V_{[1]}(\widetilde{G})$. If $t=1$, then $G=\widetilde{G}\left[x_{1}=x_{2}\right] H_{s+1}$, where $x_{1}$ and $x_{2}$ is the copy of $a$ in $\widetilde{G}$ and $H_{s+1}$, respectively, and $G^{2}$ contains hamiltonian cycle $C$ such that exactly one edge of $C$ incident with $a$ is in $G$ by Theorem $2.2 \mathrm{Ib})$. Let $t=2$. If $G_{1}=\widetilde{G}\left[x_{1}=x_{2}\right] H_{s+1}$, where $x_{1}$ and $x_{2}$ is the copy of $a$ in $\widetilde{G}$ and $H_{s+1}$, respectively, then $\left(G_{1}\right)^{2}$ is hamiltonian and $a \in V_{[2]}\left(G_{1}\right)$ by Theorem $2.2 \mathrm{Ib}$ ). Moreover $G=G_{1}\left[y_{1}=y_{2}\right] H_{s+2}$, where $y_{1}$ and $y_{2}$ is the copy of $a$ in $G_{1}$ and $H_{s+2}$, respectively, and $G^{2}$ contains hamiltonian cycle $C$ such that no edge of $C$ incident with $a$ is in $G$ by Theorem 2.2 Id).

Case 2: $r \geq 1$.

For $i=1,2, \ldots, r$, let $b_{i}$ be the branch of $\mathrm{Bl}(G)$ containing the vertex corresponding to $B_{i}$. We denote $H_{i}$ the subgraph corresponding to $b_{i}$. The block graph $\mathrm{Bl}\left(H_{i}\right)$ is a path and therefore $\left(H_{i}\right)^{2}$ is hamiltonian and $a \in V_{[1]}\left(H_{i}\right)$ either by Theorem 3.3 or by Theorem 2.1. Let $b_{r+s+1}, b_{r+s+2}$ be the branch of $\mathrm{Bl}(G)$ containing the vertex corresponding to $B_{r+s+1}$, $B_{r+s+2}$ and denote $H_{r+s+1}, H_{r+s+2}$ the subgraph corresponding to $b_{r+s+1}, b_{r+s+2}$, respectively. For $j=r+s+1, r+s+2, \operatorname{Bl}\left(H_{j}\right)$ is a path and therefore $\left(H_{j}\right)^{2}$ is hamiltonian and $a \in V_{[2]}\left(H_{j}\right)$ by Theorem 3.3.

Let $G_{1}=G-V\left(H_{r+s+1}-a\right)-V\left(H_{r+s+2}-a\right)$ and let $\ell$ denote the number of branches of $\operatorname{Bl}\left(G_{1}\right)$. We show that $\left(G_{1}\right)^{2}$ is hamiltonian and $a \in V_{[1]}\left(G_{1}\right)$.

We proceed by induction on $\ell$.

1. For $\ell=1$ obviously $G_{1}=H_{1}$ and the assertion is true.

2. Suppose the assertion is true for each graph such that its block graph contains at most $\ell$ branches, let $G_{1}$ be a graph without acyclic non-end blocks such that its block graph contains $\ell+1$ branches and with exactly one vertex in $V_{\geq 3}\left(B l\left(G_{1}\right)\right)$ corresponding to a cut vertex $a$ of $G_{1}$.

If $\widetilde{G_{1}}=G_{1}-V\left(H_{1}-a\right)$, then $G_{1}=H_{1}\left[x_{1}=x_{2}\right] \widetilde{G_{1}}$, where $x_{1}$ and $x_{2}$ is the copy of $a$ in $H_{1}$ and $\widetilde{G_{1}}$, respectively. If $\widetilde{G_{1}}=B_{r+1}$, then $\left(G_{1}\right)^{2}$ is hamiltonian and $a \in V_{[1]}\left(G_{1}\right)$ by Theorem $2.2 \mathrm{IIa})$. Otherwise $\widetilde{G_{1}}$ is hamiltonian and $a=x_{1} \in V_{[1]}\left(\widetilde{G_{1}}\right)$ by the induction hypothesis. Then $\left(G_{1}\right)^{2}$ is hamiltonian and $a \in V_{[1]}\left(G_{1}\right)$ by Theorem 2.2 Ia).

If $G=G_{1}$, then it is obvious that $G^{2}$ contains a hamiltonian cycle $C$ such that both edges of $C$ incident with $a$ are in $G$. If $t=1$, then $G=G_{1}\left[y_{1}=y_{2}\right] H_{r+s+1}$, where $y_{1}$ and $y_{2}$ is the copy of $a$ in $G_{1}$ and $H_{r+s+1}$, respectively, and $G^{2}$ contains hamiltonian cycle $C$ such that exactly one edge of $C$ incident with $a$ is in $G$ by Theorem $2.2 \mathrm{Ib}$ ). Let $t=2$. If $G_{2}=G_{1}\left[y_{1}=y_{2}\right] H_{r+s+1}$, where $y_{1}$ and $y_{2}$ is the copy of $a$ in $G_{1}$ and $H_{r+s+1}$, respectively, then $\left(G_{2}\right)^{2}$ is hamiltonian and $a \in V_{[2]}\left(G_{2}\right)$ by Theorem $\left.2.2 \mathrm{Ib}\right)$. Moreover $G=G_{2}\left[z_{1}=z_{2}\right] H_{r+s+2}$, where $z_{1}$ and $z_{2}$ is the copy of $a$ in $G_{2}$ and $H_{r+s+2}$, respectively, and $G^{2}$ contains hamiltonian cycle $C$ such that no edge of $C$ incident with $a$ is in $G$ by 
Theorem $2.2 \mathrm{Id})$.

Now we prove our main result.

Theorem 4.2. Let $G$ be a connected graph with at least three vertices such that

i) every vertex $x \in V_{\geq 3}(B l(G))$ corresponds to a cut vertex of $G$, and

ii) for any two vertices $x, y \in V_{\geq 3}(B l(G))$ it holds that $\operatorname{dist}_{B l_{(G)}}(x, y) \geq 4$.

Then $G^{2}$ is hamiltonian if and only if every cut vertex of $G$ is contained in at most two acyclic non-end blocks of $G$.

Proof. I) First suppose that we have a vertex $a \in V(G)$ contained in at least three acyclic non-end blocks of $G$. We show that the graph $G^{2}$ is not hamiltonian. Let, to the contrary, $C$ be a hamiltonian cycle in $G^{2}$. For $i=1,2,3$, let $B_{i}=a_{i} a$ denote three acyclic non-end blocks of $G$ and $B_{i+3}$ a block of $G$ adjacent to the block $B_{i}$ such that $a \notin V\left(B_{i+3}\right)$. Then necessarily there is a vertex $c_{i} \in N_{B_{i+3}}\left(a_{i}\right)$ such that $c_{i} a \in E(C)$, for $i=1,2,3$. From this it follows that $d_{C}(a) \geq 3$, contradicting the fact that $C$ is a cycle.

II) Suppose that every cut vertex of $G$ is contained in at most two acyclic non-end blocks of $G$. We show that $G^{2}$ is hamiltonian.

If $G$ is a cyclic block, then $G^{2}$ is hamiltonian by Theorem 2.1, and if $\operatorname{Bl}(G)$ is a path, then $G^{2}$ is hamiltonian by Theorem 3.2.

Now suppose that $\operatorname{Bl}(G)$ contains at least one vertex of degree at least three corresponding to a cut vertex of $G$. For $i=1,2, \ldots, k$, let $b_{i}$ be a vertex of $\mathrm{Bl}(G)$ in $V_{\geq 3}(\mathrm{Bl}(G))$, let $a_{i}$ be the vertex of $G$ corresponding to $b_{i}$ and we choose the notation such that $\operatorname{dist}_{B l(G)}\left(b_{1}, b_{k}\right)$ is maximum and the (unique) path in $\mathrm{Bl}(G)$ joining $b_{1}$ and $b_{2}$ has no interior vertices in $V_{\geq 3}(\mathrm{Bl}(G))$. Let $t_{G}\left(a_{i}\right) \geq 0$ denote the number of acyclic non-end blocks of $G$ containing $a_{i}$.

We prove the following statement.

Under the assumptions of Theorem 4.2 the graph $G^{2}$ contains a hamiltonian cycle $C$ such that if $t_{G}\left(a_{i}\right)=0$, then both edges of $C$ incident with $a_{i}$ are in $G$, if $t_{G}\left(a_{i}\right)=1$, then exactly one edge of $C$ incident with $a_{i}$ is in $G$, if $t_{G}\left(a_{i}\right)=2$, then no edge of $C$ incident with $a_{i}$ is in $G, i=1,2, \ldots, k$.

We proceed by induction on $k$.

For $k=1$ the assertion is given by Lemma 4.1 .

Suppose the assertion is true for each graph $G^{\prime}$ such that its block graph $\operatorname{Bl}\left(G^{\prime}\right)$ has at most $k-1$ vertices in $V_{\geq 3}\left(\mathrm{Bl}\left(G^{\prime}\right)\right)$ corresponding to cut vertices of $G^{\prime}$ and these are at distance at least four in $\operatorname{Bl}\left(G^{\prime}\right)$, and let $G$ be a graph such that its block graph $\operatorname{Bl}(G)$ is a tree with $k$ vertices in $V_{\geq 3}(\mathrm{Bl}(G))$ corresponding to cut vertices of $G$ and any two vertices of $\mathrm{Bl}(G)$ in $V_{>3}(\mathrm{Bl}(G))$ are at distance at least four in $\mathrm{Bl}(G), k \geq 2$.

By the notation of $a_{1}$, let $H$ be the unique subgraph of graph $G$ corresponding to the $\left(b_{1}, b_{2}\right)$-path in $\operatorname{Bl}(G)$. Let $\widetilde{G}=G-V\left(H-\left\{a_{1}, a_{2}\right\}\right)$. We denote the components of $\widetilde{G}$ by $G_{1}, G_{2}$ such that $a_{1} \in V\left(G_{1}\right)$ and $a_{2} \in V\left(G_{2}\right)$. 
If $d_{\mathrm{Bl}_{\left(G_{1}\right)}}\left(a_{1}\right) \geq 3$ and $d_{\mathrm{Bl}_{\left(G_{2}\right)}}\left(a_{2}\right) \geq 3$, then, by the induction hypothesis, $\left(G_{1}\right)^{2},\left(G_{2}\right)^{2}$ contains a hamiltonian cycle $C_{1}, C_{2}$ such that if $t_{G_{1}}\left(a_{1}\right)=0, t_{G_{2}}\left(a_{i}\right)=0$, then both edges of $C_{1}, C_{2}$ incident with $a_{1}, a_{i}$ are in $G_{1}, G_{2}$, if $t_{G_{1}}\left(a_{1}\right)=1, t_{G_{2}}\left(a_{i}\right)=1$, then exactly one edge of $C_{1}, C_{2}$ incident with $a_{1}, a_{i}$ is in $G_{1}, G_{2}$, if $t_{G_{1}}\left(a_{1}\right)=2, t_{G_{2}}\left(a_{i}\right)=2$, then no edge of $C_{1}, C_{2}$ incident with $a_{1}, a_{i}$ is in $G_{1}, G_{2}, i=2,3, \ldots, k$, respectively.

In the case $d_{\mathrm{Bl}_{\left(G_{1}\right)}}\left(a_{1}\right)=2$, set $K_{2}=v u$ (where $v, u \notin V(G)$ ), and $\widehat{G_{1}}=G_{1}\left[a_{1}=v\right] K_{2}$. Then $\left(\widehat{G_{1}}\right)^{2}$ contains a hamiltonian cycle with the required properties by the induction hypothesis and it is obvious that also $\left(G_{1}\right)^{2}$. We proceed in the case $d_{\operatorname{Bl}\left(G_{2}\right)}\left(a_{2}\right)=2$ similarly.

Then by the assumption that any two vertices of $\mathrm{Bl}(G)$ in $V_{\geq 3}(\mathrm{Bl}(G))$ are at distance at least four in $\operatorname{Bl}(G)$ and by Theorem 3.3, the graph $H^{2}$ contains a hamiltonian cycle $C_{H}$ such that, for $j=1,2$, if $a_{j}$ is contained in a cyclic block, then both edges of $C_{H}$ incident with $a_{j}$ are in $H$, and if $a_{j}$ is contained in an acyclic block, then exactly one edge of $C_{H}$ incident with $a_{j}$ is in $H$.

Case 1: $t_{G_{2}}\left(a_{2}\right) \in\{0,1\}$.

Let $\widetilde{G_{2}}=G_{2}\left[x_{1}=x_{2}\right] H$, where $x_{1}$ and $x_{2}$ is the copy of $a_{2}$ in $G_{2}$ and $H$, respectively. Then $\left(\widetilde{G_{2}}\right)^{2}$ contains a hamiltonian cycle $\widetilde{C_{2}}$ with the required properties by Theorem 2.2 either $\mathrm{Ia}$ ) or $\mathrm{Ib}$ ) or Id) (using $C_{H}$ and $C_{2}$ ). Moreover it is obvious that if $a_{1}$ is contained in a cyclic block of $\widetilde{G_{2}}$, then both edges of $\widetilde{C_{2}}$ incident with $a_{1}$ are in $\widetilde{G_{2}}$, and if $a_{1}$ is contained in an acyclic block of $\widetilde{G_{2}}$, then exactly one edge of $\widetilde{C_{2}}$ incident with $a_{1}$ is in $\widetilde{G_{2}}$.

a) If $t_{G_{1}}\left(a_{1}\right) \in\{0,1\}$, then $G=G_{1}\left[y_{1}=y_{2}\right] \widetilde{G_{2}}$, where $y_{1}$ and $y_{2}$ is the copy of $a_{1}$ in $G_{1}$ and $\widetilde{G_{2}}$, respectively, and $G^{2}$ contains a hamiltonian cycle $C$ such that if $t_{G}\left(a_{i}\right)=0$, then both edges of $C$ incident with $a_{i}$ are in $G$, if $t_{G}\left(a_{i}\right)=1$, then exactly one edge of $C$ incident with $a_{i}$ is in $G$, if $t_{G}\left(a_{i}\right)=2$, then no edge of $C$ incident with $a_{i}$ is in $G, i=1,2, \ldots, k$, by Theorem 2.2 either Ia) or Ib) or Id) (using $\widetilde{C_{2}}$ and $C_{1}$ ).

b) Let $t_{G_{1}}\left(a_{1}\right)=2$. Let $B_{1}$ be an acyclic non-end block of $G_{1}$ containing the vertex $a_{1}$, let $F$ be the subgraph of $G_{1}$ corresponding to the maximal connected subgraph of $\mathrm{Bl}\left(G_{1}\right)$ containing the vertex corresponding to $B_{1}$ and not containing the vertex $b_{1}$. Let $F_{1}=G_{1}-V\left(F-a_{1}\right)$. By the induction hypothesis, $G_{1}$ contains a hamiltonian cycle $C_{1}$ such that no edge of $C_{1}$ incident with $a_{1}$ is in $G_{1}$ and we can divide $C_{1}$ into hamiltonian cycles $C_{1 a}$ in $F_{1}$ and $C_{1 b}$ in $F$ such that exactly one edge of $C_{1 a}$ incident with $a_{1}$ is in $F_{1}$ and exactly one edge of $C_{1 b}$ incident with $a_{1}$ is in $F$.

Necessarily both edges of $\widetilde{C_{2}}$ incident with $a_{1}$ are in $\widetilde{G_{2}}$ (otherwise $t_{G}\left(a_{1}\right)=3$, a contradiction). Set $\widetilde{G_{1}}=F_{1}\left[y_{1}=y_{2}\right] \widetilde{G_{2}}$, where $y_{1}$ and $y_{2}$ is the copy of $a_{1}$ in $F_{1}$ and $\widetilde{G_{2}}$, respectively. Then $\left(\widetilde{G_{1}}\right)^{2}$ contains a hamiltonian cycle $\widetilde{C_{1}}$ with the required properties by Theorem $2.2 \mathrm{Ib}$ ) (using $C_{1 a}$ and $\widetilde{C_{2}}$ ).

Then $G=\widetilde{G_{1}}\left[z_{1}=z_{2}\right] F$, where $z_{1}$ and $z_{2}$ is the copy of $a_{1}$ in $\widetilde{G_{1}}$ and $F$, respectively, and $G^{2}$ contains a hamiltonian cycle $C$ such that if $t_{G}\left(a_{i}\right)=0$, then both edges of $C$ incident with $a_{i}$ are in $G$, if $t_{G}\left(a_{i}\right)=1$, then exactly one edge of $C$ incident with 
$a_{i}$ is in $G$, if $t_{G}\left(a_{i}\right)=2$, then no edge of $C$ incident with $a_{i}$ is in $G, i=1,2, \ldots, k$, by Theorem $2.2 \mathrm{Id}$ ) (using $\widetilde{C_{1}}$ and $C_{1 b}$ ).

Case 2: $t_{G_{2}}\left(a_{2}\right)=2$.

Let $B_{2}$ be an acyclic non-end block of $G_{2}$ containing the vertex $a_{2}$, let $S$ be the subgraph of $G_{2}$ corresponding to the maximal connected subgraph of $\operatorname{Bl}\left(G_{2}\right)$ containing the vertex corresponding to $B_{2}$ and not containing the vertex $b_{2}$.

Let $S_{1}=G_{2}-V\left(S-a_{2}\right)$. By the induction hypothesis, $G_{2}$ contains a hamiltonian cycle $C_{2}$ such that no edge of $C_{2}$ incident with $a_{2}$ is in $G$ and we can divide $C_{2}$ into hamiltonian cycles $C_{2 a}$ in $S_{1}$ and $C_{2 b}$ in $S$ such that exactly one edge of $C_{2 a}$ incident with $a_{2}$ is in $S_{1}$ and exactly one edge of $C_{2 b}$ incident with $a_{2}$ is in $S$.

Now necessarily both edges of $C_{H}$ incident with $a_{2}$ are in $H$ (otherwise $t_{G}\left(a_{2}\right)=3$, a contradiction). Set $\widetilde{S}_{1}=S_{1}\left[x_{1}=x_{2}\right] H$, where $x_{1}$ and $x_{2}$ is the copy of $a_{2}$ in $S_{1}$ and $H$, respectively. Then $\left(\widetilde{S}_{1}\right)^{2}$ contains a hamiltonian cycle $C^{\prime}$ with the required properties by Theorem $2.2 \mathrm{Ib}$ ) (using $C_{2 a}$ and $C_{H}$ ).

Then $\widetilde{G_{2}}=\widetilde{S}_{1}\left[u_{1}=u_{2}\right] S$, where $u_{1}$ and $u_{2}$ is the copy of $a_{2}$ in $\widetilde{S}_{1}$ and $S$, respectively, and $\left(\widetilde{G_{2}}\right)^{2}$ contains a hamiltonian cycle $\widetilde{C_{2}}$ with the required properties by Theorem $\left.2.2 \mathrm{Id}\right)$ (using $C^{\prime}$ and $C_{2 b}$ ). Moreover it is obvious that if $a_{1}$ is contained in a cyclic block of $\widetilde{G_{2}}$, then both edges of $\widetilde{C_{2}}$ incident with $a_{1}$ are in $\widetilde{G_{2}}$, and if $a_{1}$ is contained in an acyclic block

of $\widetilde{G_{2}}$, then exactly one edge of $\widetilde{C_{2}}$ incident with $a_{1}$ is in $\widetilde{G_{2}}$. Then we continue similarly as in Subcase 1a) or 1b).

It is obvious that the conditions in Theorem 4.2 can be verified in polynomial time. From this it follows that the decision problem, if the square of a graph is hamiltonian, which is NP-complete in general ([3]), can be decided in polynomial time in the class of the graphs $G$ such that every vertex $x \in V_{\geq 3}(\mathrm{Bl}(G))$ corresponds to a cut vertex of $G$, and for any two vertices $x, y \in V_{\geq 3}(\mathrm{Bl}(G))$ it holds that $\operatorname{dist}_{\mathrm{Bl}_{(G)}}(x, y) \geq 4$.

The following theorems are immediate corollaries of Theorem 4.2.

Corollary 4.3. Let $G$ be a connected graph such that its block graph $\mathrm{Bl}(G)$ is homeomorphic to a star in which the center corresponds to a cut vertex a of $G$. Then the graph $G^{2}$ is hamiltonian if and only if the vertex $a$ is contained in at most two acyclic non-end blocks of $G$.

Corollary 4.4. If the block graph of $G$ with at least three vertices is a star, then $G^{2}$ is hamiltonian.

Note that the graph in Figure 1 satisfies the assumptions of Corollary 4.3. Therefore Corollary 4.3 (hence also Theorem 4.2) does not follow from Theorem 3.1. 


\section{A star in which the center corresponding to a block}

Let $G$ be a connected graph such that its block graph $\mathrm{Bl}(G)$ is homeomorphic to a star in which the center corresponds to a block $B_{c}$ of $G$. If $B_{c}$ is acyclic, then $\operatorname{Bl}(G)$ is a path and $G^{2}$ is hamiltonian by Theorem 3.2.

Let $B_{c}$ be cyclic. Let $k$ denote the number of cut vertices of $G$ in $V\left(B_{c}\right)$, let $v_{i} \in V\left(B_{c}\right)$ be all cut vertices of $G$ in $B_{c}, i=1,2, \ldots, k$. Let $C$ be a hamiltonian cycle in $\left(B_{c}\right)^{2}$. We say that $C$ is acceptable in $G$ if there are pairwise distinct edges $v_{i} w_{i} \in E(C)$ such that $v_{i} w_{i} \in E\left(B_{c}\right)$, for any $i=1,2, \ldots, k$.

The following theorem gives only a sufficient condition for hamiltonicity in this class of graphs (in comparison with Theorem 4.2).

Theorem 5.1. Let $G$ be a connected graph such that its block graph $\mathrm{Bl}(G)$ is homeomorphic to a star in which the center corresponds to a block $B_{c}$ of $G$. If $\left(B_{c}\right)^{2}$ contains an acceptable cycle in $G$, then $G^{2}$ is hamiltonian.

Proof. Let $v_{i} \in V\left(B_{c}\right)$ be all cut vertices of $G$ in $V\left(B_{c}\right), i=1,2, \ldots, k$. We prove the following slight strengthening of Theorem 5.1.

Let $G$ be a connected graph such that its block graph $B l(G)$ is homeomorphic to a star in which the center corresponds to a block $B_{c}$ of $G$. If $\left(B_{c}\right)^{2}$ contains an acceptable cycle $C$ in $G$, then $G^{2}$ contains a hamiltonian cycle containing all edges of $C$ except the edges $v_{i} w_{i}$, $i=1,2, \ldots, k$.

We prove this assertion by induction on $k$.

1. Let $k=0$. Then $G=B_{c}$ and the assertion is true.

2. Suppose the assertion is true for each graph such that the block $B_{c}$ contains at most $k-1$ cut vertices, let $G$ be a graph such that its block graph is homeomorphic to a star in which the center corresponds to a block $B_{c}$ of $G$ and $B_{c}$ contains $k$ cut vertices of $G$, $k \geq 1$.

Let $d \in V(\operatorname{Bl}(G))$ be the vertex corresponding to $B_{c}$, let $G^{\prime}$ be the subgraph of $G$ such that $\operatorname{Bl}\left(G^{\prime}\right)=\operatorname{Bl}(G)-d$ and let $H_{i}$ be the component of $G^{\prime}$ such that $v_{i} \in V\left(H_{i}\right)$, for $i=1,2, \ldots, k$. The block graph $\operatorname{Bl}\left(H_{i}\right)$ is a path and therefore either $\left(H_{i}\right)^{2}$ is hamiltonian and $v_{i} \in V_{[1]}\left(H_{i}\right) \cup V_{[2]}\left(H_{i}\right)$ (either by Theorem 3.3 or by Theorem 2.1) or $H_{i}$ is isomorphic to one edge.

Let $G_{1}=G-V\left(H_{1}-v_{1}\right)$ and let $x_{1}$ and $x_{2}$ denote the copy of $v_{1}$ in $G_{1}$ and $H_{1}$, respectively. Then $G=G_{1}\left[x_{1}=x_{2}\right] H_{1}$. Let $C$ be an acceptable cycle in $G$. Then $C$ is also acceptable in $G_{1}$ and therefore $\left(G_{1}\right)^{2}$ contains a hamiltonian cycle $C_{1}$ containing all edges of $C$ except $v_{i} w_{i}, i=2,3, \ldots, k$, by the induction hypothesis. The cycle $C$ is acceptable in $G$ and therefore $x_{1} w_{1} \in E\left(G_{1}\right)$ and $x_{1} w_{1} \in E\left(C_{1}\right)$. Then $x_{1} \in V_{[1]}\left(G_{1}\right) \cup V_{[2]}\left(G_{1}\right)$.

Case 1: $x_{2} \in V_{[1]}\left(H_{1}\right) \cup V_{[2]}\left(H_{1}\right)$.

Then $G^{2}$ contains a hamiltonian cycle containing all edges of $C$ except $v_{i} w_{i}, i=1,2, \ldots, k$, by Theorem $2.2 \mathrm{I}$ ).

Case 2: $H_{1}$ is isomorphic to one edge. 
Then $G^{2}$ contains a hamiltonian cycle containing all edges of $C$ except $v_{i} w_{i}, i=1,2, \ldots, k$, by Theorem 2.2 either IIa) or IIb).

The following theorem is an immediate corollary of Theorem 5.1.

Corollary 5.2. Let $G$ be a connected graph such that its block graph $\mathrm{Bl}(G)$ is homeomorphic to a star in which the center corresponds to a block $B_{c}$ of $G$. If $B_{c}$ is hamiltonian, then $G^{2}$ is hamiltonian.

Let us mention the following theorem by Schaar [4] that motivates the next conjecture.

Theorem $5.3[4]$. For every block $G$ with $|V(G)| \geq 4$ there exists a hamiltonian cycle in $G^{2}$ containing at least four edges of $G$.

Conjecture 5.4. Let $G$ be a connected graph such that its block graph $B l(G)$ is homeomorphic to a star in which the center c corresponds to a block $B_{c}$ of $G$. If $d_{B l_{(G)}}(c) \leq k$, $k<5$, then $G^{2}$ is hamiltonian.

Conjecture 5.4 is true for $k \leq 2$ but for $k \in\{3,4\}$ is an open problem. It is not possible to specify four edges from Theorem 5.3 and therefore Conjecture 5.4 is not an immediate corollary of Theorem 5.3. If Conjecture 5.4 is true, then the upper bound is sharp as can be seen from Figure 2.

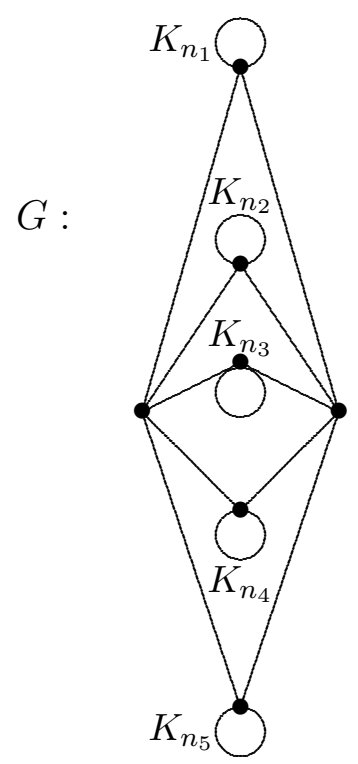

Figure 2: 


\section{Conclusion and acknowledgement}

In conclusion I would like to thank Z. Ryjáček for valuable notes and recommendations.

\section{References}

[1] M. El Kadi Abderrezzak, E. Flandrin, Z. Ryjáček: Induced $S\left(K_{1,3}\right)$ and hamiltonian cycles in the square of a graph, Discrete Mathematics 207 (1999), 263-269.

[2] H. Fleischner: In the square of graphs, hamiltonicity and pancyclity, hamiltonian connectedness and panconnectedness are equivalent concepts, Monatshefte für Mathematik 82 (1976), 125-149.

[3] P. Underground: On graphs with hamiltonian squares, Discrete Mathematics 21 (1978), 323.

[4] G. Schaar: On 'maximal' Hamiltonian cycles in the square of a block, Discrete Mathematics 121 (1993), 195-198.

[5] C. Thomassen: The square of a graph is hamiltonian provided its block graph is a path, preprint, unpublished. 